\title{
Volcanic ash decreases dehydration tolerance in the field cricket Gryllus bimaculatus (Orthoptera: Gryllidae)
}

\section{Authors: Takashi Kuriwada \& Gadi V.P. Reddy}

NOTICE: this is the author's version of a work that was accepted for publication in Journal of Asia-Pacific Entomology. Changes resulting from the publishing process, such as peer review, editing, corrections, structural formatting, and other quality control mechanisms may not be reflected in this document. Changes may have been made to this work since it was submitted for publication. A definitive version was subsequently published in Journal of Asia-Pacific

Entomology, [VOL\# 19, ISSUE\# 1, (March 2016)] DOI\#10.1016/j.aspen.2015.12.005

Kuriwada, Takashi, and Gadi V.P. Reddy. "Volcanic ash decreases dehydration tolerance in the field cricket Gryllus bimaculatus (Orthoptera: Gryllidae)." Journal of Asia-Pacific Entomology 19, no. 1 (March 2016): 85-87. DOI:https://dx.doi.org/10.1016/j.aspen.2015.12.005.

Made available through Montana State University's $\underline{\text { ScholarWorks }}$ scholarworks. montana.edu 


\title{
Volcanic ash decreases dehydration tolerance in the field cricket Gryllus bimaculatus (Orthoptera: Gryllidae)
}

\author{
Takashi Kuriwada ${ }^{\mathrm{a}}$ \& Gadi V.P. Reddy \\ ${ }^{a}$ Faculty of Education, Laboratory of Zoology, Kagoshima University, Korimoto 1-20-6, Kagoshima 890-0065, Japan \\ ${ }^{\mathrm{b}}$ Western Triangle Agricultural Research Center,Montana State University, 9546 Old Shelby Rd., P. O. Box 656, Conrad, MT 59425, \\ USA
}

\begin{abstract}
Volcanic eruptions have catastrophic impacts on ecosystems, ranging in scale from effects on individual behavior to changes in community structure. Volcanic ash has an especially widespread effect, as it can be dispersed over a greater distance than other volcanic products. In this study, we examined the effect of volcanic ash on the survival and dehydration tolerance of the field cricket Gryllus bimaculatus De Geer (Orthoptera: Gryllidae). Although nymphal mortality, developmental period, and adult body size were not affected by volcanic ash, the dehydration tolerance (lifespan without water) of adults reared on a substrate of volcanic ash was lower than that of adults reared on vermiculite. This suggests that volcanic ash may decrease the fitness of field crickets, depending on the availability of water.
\end{abstract}

\section{Introduction}

Disturbances of a global scale such as volcanic eruptions can change environments rapidly, affecting individuals, populations, communities, and ecosystems. In addition to the direct effect of lava flow and volcanic rock, ash released by volcanic eruptions can disperse over longer distances than other volcanic products such as lava and pumice, influencing both the behavior of individuals and the structure of biological communities (Wilson et al., 2011). Because volcanic ash contains glass shards $\left(\mathrm{SiO}_{2}\right)$ and alumina $\left(\mathrm{Al}_{2} \mathrm{O}_{3}\right)$ (Shirakawa et al., 1984), animal bodies are often injured by the sharp ash particles (Edwards and Schwartz, 1981).

Such physical features cause a variety of effects on fitness components of wild animals. For example, volcanic ash has been found to affect the locomotor performance of the lizard Phymaturus spectabilis Lobo and Quinteros (Cabezas-Cartes et al., 2014). Edwards and Schwartz (1981) showed that water loss by cuticular abrasion by ash and excessive salivation during grooming increased mortality rates of house cricket Acheta domesticus and cockroach Periplaneta americana. Shanks and Chase (1981) reported that placing ash on leaves inhibited feeding by herbivorous insects, while Marske (2004) found certain taxa to be more susceptible than others. Furthermore, volcanic ash affects inter-specific interactions. For example, bee fauna and plant-pollinator interactions were negatively affected by volcanic ash in the 2011 eruption of the Puyehue-Cordón Caulle volcanic complex in Patagonia (Morales et al., 2014). They found a significant decrease in total bee richness visiting raspberry flowers and the number of bumble bees visiting wild flowers (Morales et al., 2014).

Mt. Sakurajima is an active volcano in Japan which undergoes frequent small-scale eruptions (e.g. 1107 eruptions in 2012, 1097 eruptions in 2013, and 656 eruptions in 2014: Kagoshima Meteorological Office). Volcanic ash from Mt. Sakurajima is carried by the wind to fall over a downwind swath of land $\left(3602 \mathrm{~g} / \mathrm{m}^{2}\right.$ in $2012,1833 \mathrm{~g} / \mathrm{m}^{2}$ in 2013, and $995 \mathrm{~g} / \mathrm{m}^{2}$ in 2014, measured at Higashi Korimoto, Kagoshima city: Kagoshima Meteorological Office). The fallen ash attaches to the surfaces of plants and insect bodies (Fig. 1). The volcanic ash with the sharp ash particles will lead to reduced survival, growth, and dehydration tolerance due to increased water loss through the damaged cuticle (Edwards and Schwartz, 1981). Indeed, the cuticle beneath the lipid layer protects from desiccation in ants Solenopsis invicta and S. richteri (Chen et al., 2014).

Laboratory tests conducted by Fernández-Arhex et al. (2013) found that constant exposure to volcanic ash and diatomaceous earth induces mortality in adults of the grasshopper Dichroplus vittigerum (Blanchard) (Orthoptera: Acrididae), similar to that produced by diatomaceous earth. Field crickets typically live in grassy fields, and thus are likely to suffer considerable exposure to volcanic ash. However, cricket populations with historical exposure to volcanic ash may have adapted to it. Consequently, in these experiments, Gryllus bimaculatus (De Geer), a species from areas not exposed to volcanic ash, was used to test the potential effects of volcanic ash. Using this naive species, we examined the effects of volcano ash on cricket mortality, developmental period, and dehydration tolerance. 


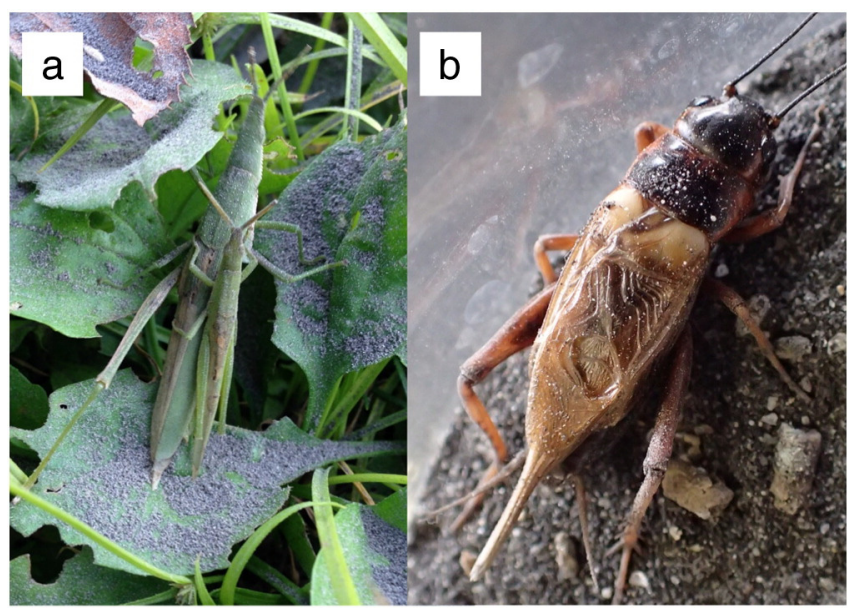

Fig. 1. (a) Volcanic ash accumulation on the grasshopper Atractomorpha lata (Mochulsky) in the field conditions after the eruption of the Sakurajima volcano. (b) Volcanic ash accumulation on the field cricket of the present study.

\section{Materials and methods}

Source and rearing of insects

All individuals used in this experiment were fifth-generation progeny of crickets purchased from a local pet store. Approximately 300 adult crickets were reared in plastic breeding containers $(58.0 \times 38.5 \times 31.5 \mathrm{~cm})$ at $25 \pm 1{ }^{\circ} \mathrm{C}$ and at $14: 10 \mathrm{~h}(\mathrm{~L}: \mathrm{D}$ photoperiod (light cycle: $0200-1600$ h). Relative humidity was not controlled throughout our experiment (range ca. 60-80\%). However, all experimental treatments were conducted at a time. Therefore, all focal crickets were experienced in the range of humidity in the similar way. The crickets were supplied with egg-cartons for shelter, moist sand in a 500-mL plastic cup as a water source, and an excess of insect food pellets (Oriental Koubo, Tokyo, Japan). The sand was sprinkled with water every 2-3 days. The study was conducted from November 2014 to January 2015.

\section{Experiment 1: Effect of substrate on nymphal attributes}

Two weeks after hatching, nymphs were collected from the breeding tank and randomly assigned to one of three cage-floor materials as treatments: Akadama soil (i.e., commercial garden soil), vermiculite, or volcanic ash. (Akadama soil is formed from accumulated volcanic ash through weathering, which smooths the sharp-angled edges of fresh ash particles.) Nymphs were placed on substrates in plastic containers $(29.9 \times 19.2 \times 20.1 \mathrm{~cm})$ in groups of 30 and held until adult emergence. Each treatment was replicated five times, for a total of 150 nymphs per substrate. All test containers contained wet cotton in a Petri dish ( $9 \mathrm{~cm}$ diameter; $2 \mathrm{~cm}$ height), excess food, and egg carton shelters. Newly emerged adults were collected and counted weekly, and their developmental time (weekly checked) and body weight (eclosion) was noted. Young adults of body integrity (aged 1-6 days) were then used in subsequent experiments, as described below.

\section{Experiment 2: Dehydration tolerance}

Body weights of young adults (age 1-6 days) obtained from the substrate experiment described above were measured (to the nearest $0.1 \mathrm{mg}$ ) using an electronic balance (Mettler AE50 balance). Adult crickets were then either maintained on the same floor materials as during their nymphal life or were held on filter paper as a floor material, for total of six treatment groups. Adult crickets were held individually in plastic cups (10 cm diameter; $3 \mathrm{~cm}$ height) with ad libitum dry pellets for insects (water content was ca 7\%: Oriental Koubo, Tokyo, Japan) and no water source and recorded as dead or alive daily.

\section{Statistical analyses}

We used generalized linear mixed models (GLMMs) (Bolker et al., 2009) to test for effects of floor materials on mortality rate (binomial error and logit link function), developmental time (Poisson error and identity log function), and body weight (Gaussian error and identity link function), by using containers as replicates (i.e., random effect). GLMMs with Poisson error and logit link function were conducted to examine the effect of floor materials on dehydration tolerance of adults. A likelihood ratio test was used to determine the statistical significance of each coefficient in the models. Tukey's multiple comparisons (R addon package multcomp) (Hothorn et al., 2008) were used to compare among treatment groups. All statistical calculations were performed using R version 3.0.3 (R Core Team, 2014).

\section{Results}

\section{Experiment 1: Effect of substrate on nymphal attributes}

We found that the type of floor material in cages had no significant effect on any attribute examined, including nymphal mortality rate (Table $1 ; \chi^{2}=4.62$, d.f. $=2, \mathrm{p}=0.099$ ), nymphal developmental period (Table. $1 ; \chi^{2}=2.45$, d.f. $=2, \mathrm{p}=0.29$ ), or young adult body weight $\left(\chi^{2}=1.36\right.$, d.f. $\left.=2, \mathrm{p}=0.51\right)$. The developmental period of male nymphs was longer than that of females (Table $1 ; \chi^{2}=21.17$, d.f. $=$ $1, \mathrm{p}<0.0001)$. Body weights of young adult males were heavier than those of females (Table. $1 ; \chi^{2}=6.08$, d.f. $=1, \mathrm{p}=0.014$ ), and adults with longer developmental times had heavier body weights $(\beta=$ $6.70 \pm 1.39, \chi^{2}=22.44$, d.f. $=1, \mathrm{p}<0.0001$ ) for both sexes.

\section{Dehydration tolerance}

For adults reared on the same floor material as during their nymphal stage, the dehydration tolerance (i.e., lifespan with no water source) of crickets on the Akadama soil and volcano ash was lower than that of those reared on vermiculite (Fig. 2a). We found no significant difference between sexes $\left(\chi^{2}=0.54\right.$, d.f. $\left.=1, p=0.46\right)$, but heavier crickets had a significantly higher dehydration tolerance $\left(\beta=0.0041 \pm 0.0014, \chi^{2}=\right.$ 8.54 , d.f. $=1, \mathrm{p}=0.0035)$. For crickets reared as adults on filter paper, the dehydration tolerance of crickets on the Akadama soil was higher than that of those reared on volcanic ash (Fig. 2b). We found no significant effects of $\operatorname{sex}\left(\chi^{2}=0.056\right.$, d.f. $\left.=1, \mathrm{p}=0.81\right)$ or of weight $\left(\beta=-0.00074 \pm 0.0033, \chi^{2}=0.058\right.$, d.f. $\left.=1, p=0.81\right)$ on the

Table 1

Mean \pm standard deviation of the cricket traits.

\begin{tabular}{|c|c|c|c|c|c|c|}
\hline Treatment & Survival rate & Sex & Developmental time (day) & $\mathrm{N}$ & Body weight (mg) & $\mathrm{N}$ \\
\hline Volcanic ash & $0.47 \pm 0.10$ & Male & $72.2 \pm 6.53$ & 25 & $674 \pm 136$ & 24 \\
\hline Akadama soil & $0.55 \pm 0.17$ & & $74.5 \pm 6.25$ & 36 & $695 \pm 96.3$ & 31 \\
\hline Vermiculite & $0.45 \pm 0.06$ & & $73.3 \pm 6.88$ & 30 & $696 \pm 105$ & 25 \\
\hline Volcanic ash & & Female & $67.5 \pm 7.04$ & 47 & $695 \pm 152$ & 39 \\
\hline Akadama soil & & & $69.3 \pm 6.46$ & 42 & $734 \pm 126$ & 38 \\
\hline Vermiculite & & & $66.9 \pm 5.33$ & 33 & $670 \pm 108$ & 25 \\
\hline
\end{tabular}


a

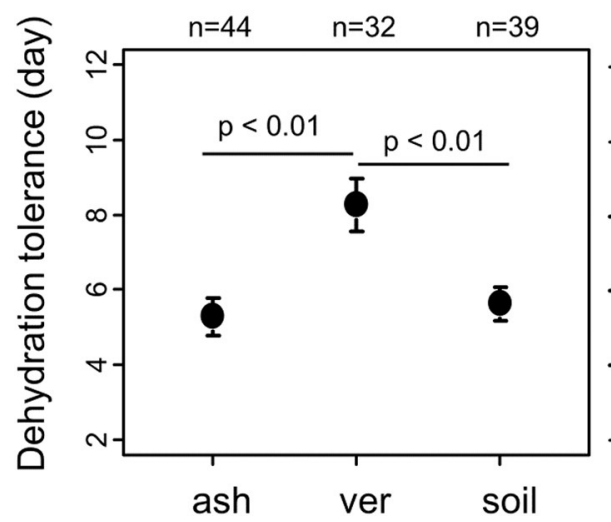

b

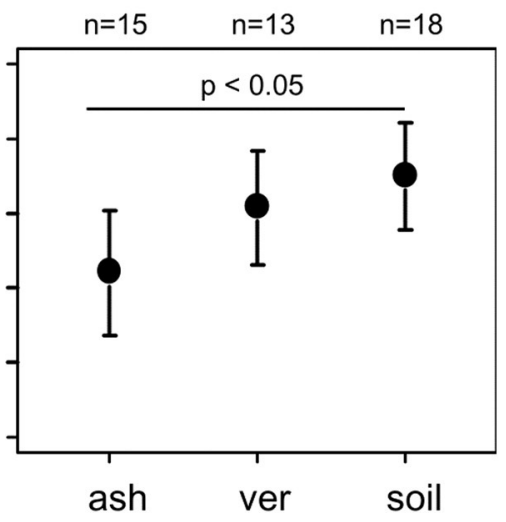

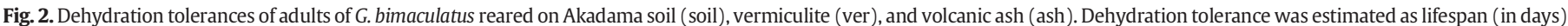

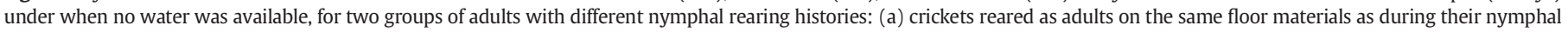
period and (b) crickets reared as adults on filter paper but derived from nymphs reared on the indicated cage substrates. Error bars indicate $95 \%$ confidence intervals.

dehydration tolerance of adult crickets when they were held on a nonabrasive floor substrate (filter paper). These results and Fig. 2ab suggested that adults reared on Akadama soil only as nymph stage exhibited higher dehydration tolerance than ones reared on soil their whole lives (i.e., 95\% confidence intervals did not overlap). On the other hand, there was no significant differences in crickets reared over paper as adult period vs crickets reared over ash for their whole lives, showing that there is some carryover damage from their nymphal experience.

\section{Discussion}

Exposure to volcanic ash had no significant effects, positive or negative, on nymphal mortality, developmental period, and or young adult body weight. Ample water was provided to the crickets through the experimental period, which may have compensated for any damage caused by ash during the experiment. To test the potential effect of volcanic ash on these fitness components in the field, water availability under field conditions should be determined and simulated. It is possible that evaporation from body abrasions may be minimal in a humid environment such as Japan. In more arid terrain, arthropods may be more strongly affected by volcanic ash.

For adults reared on the same floor materials as throughout their life as nymphs, adult dehydration tolerance on volcanic ash and Akadama soil was lower than that on vermiculite. However, adults reared on filter paper derived from nymphs reared on Akadama soil had a higher dehydration tolerance than adults reared on filter paper derived from nymphs reared on volcanic ash. This suggests that while both Akadama soil and volcanic ash reduced the dehydration tolerance of the crickets, the mechanism involved differed. While cricket dehydration tolerance (as adults) was reduced on soil only after continuous contact with Akadama soil (as nymphs and adults), adult crickets still experienced a reduction in dehydration tolerance after only nymphal exposure to volcano ash, suggesting some form of carryover effect. Because Akadama soil is porous and highly water retentive (Liu et al., 1997), dried Akadama soil may absorb moisture from its environment. If crickets are in contact with Akadama soil, dehydration tolerance might therefore be weak because the substrate acquires moisture from the surrounding environment.

In this study, we used commercially reared G. bimaculatus, with no opportunity for adaptation to volcanic ash, to detect the potential costs to cricket fitness of exposure to volcanic ash. Field cricket species in regions around volcanic mountains may exhibit adaptations to the ash, which would be an excellent direction for future study.

\section{Acknowledgments}

Dr. Tomoaki Matsui (Kagoshima University) provided useful information on Mt. Sakurajima and the composition of volcanic ash. Kazuaki Sato provided materials for rearing crickets. The English used in this manuscript was revised by Van Driesche Scientific Editing.

\section{References}

Bolker, B.M., Brooks, M.E., Clark, C.J., Geange, S.W., Poulsen, J.R., Stevens, M.H., White, J.S., 2009. Generalized linear mixed models: a practical guide for ecology and evolution. Trends Ecol. Evol. 24, 127-135.

Cabezas-Cartes, F., Kubisch, E.L., Ibargüengoytía, N.R., 2014. Consequences of volcanic ash deposition on the locomotor performance of the Phymaturus spectabilis lizard from Patagonia, Argentina. J. Exp. Zool. 321A, 164-172.

Chen, J., Rashid, T., Feng, G., 2014. A comparative study between Solenopsis invicta and Solenopsis richteri on tolerance to heat and desiccation stresses. PLoS ONE 9, e96842.

Edwards, J.S., Schwartz, L.M., 1981. Mount St. Helens ash: a natural insecticide. Can. J. Zool. $59,714-715$.

Fernández-Arhex, V., Buteler, M., Amadio, M.E., Enriquez, A., Pietrantuono, A.L., Stadler, T., Becker, G., Bruzzone, O., 2013. The effects of volcanic ash from Puyehue-Caulle range eruption on the survival of Dichroplus vittigerum (Orthoptera: Acrididae). Fla. Entomol. 96, 286-288.

Hothorn, T., Bretz, F., Westfall, P.H., 2008. Simultaneous inference in parametric models. Biom. J. 50, 346-363.

Liu, W., Peng, S.W., Mizukami, K., 1997. Moisture evaporation and migration in thin porous packed bed influenced by ambient and operating conditions. Int. J. Energy Res. 21, 41-53.

Marske, KA 2004. Effects of volcanic ash on the insect food of the Montserrat Oriole Icterus oberi Lawrence 1880 (Masters Thesis) Montana State University, Bozeman, Montana, USA (178 pp.).

Morales, C.L., Saez, A., Arbetman, M.P., Cavaleero, L., Aizen, M.A., 2014. Detrimental effects of volcanic ash deposition on bee fauna and plant-pollinator interactions. Ecol. Aust. $24,42-50$.

$\mathrm{R}$ Core Team, 2014. R: A language and environment for statistical computing. $\mathrm{R}$ Foundation for Statistical Computing, Vienna, Austria (URL http://www.Rproject.org/).

Shanks Jr., C.H., Chase, D.L., 1981. Effect of volcanic ash on adult Otiorhynchus (Coleoptera: Curculionidae). Melanderia 37, 63-66.

Shirakawa, M., Fukushima, R., Kyushima, K., 1984. Experimental studies on the effects of Mt. Sakurajima volcanic ashes on the respiratory organs. Jpn. J. Ind. Health 26, 130-146.

Wilson, T.M., Cole, J.W., Stewart, C., Cronin, S.J., Johnston, D.M., 2011. Ash storms: impacts of wind-remobilised volcanic ash on rural communities and agriculture following the 1991 Hudson eruption, southern Patagonia, Chile. Bull. Volcanol. 73, 223-239. 